\title{
Studies on Rheology, Strength and Cementing Efficiency of High Strength Grade Quaternary Blended Self-Compacting Concrete Incorporating High Reactivity Metakaolin
}

\author{
M V Seshagiri Rao ${ }^{1}$ S Shrihari ${ }^{2}$, V Srinivasa Reddy ${ }^{3}$ \\ 'CVR College of Engineering/Civil Engineering Department, Hyderabad, India. \\ Email:rao_vs_meduri@yahoo.com \\ 2JBIET/ Civil Engineering Department, Hyderabad, India. \\ Email;shriharistructure@gmail.com \\ ${ }^{3}$ GRIET/ Civil Engineering Department, Hyderabad, India. \\ Email:vempada@gmail.com
}

\begin{abstract}
The present work aims at determining the most suitable mix proportion that can produce metakaolin based quaternary blended high strength SCC of desirable strength. The results of this study will lead to the reduction of the usage of cement, further sustainable development in the concrete industry by reusing industrial waste by-products (SCMs) as cement replacements and reducing harmful impact on the environment. This study systematically investigate the synergistic effect of metakaolin (MK) and microsilica (MS) on fresh and strength properties of fly ash based SCC of M80 grade. These results are compared to establish the enhanced micro-structural and engineering properties of metakaolin based quaternary blended SCC.By incorporating MK into MS+FA based ternary blended SCC mixes, the amount of fly ash used has almost doubled. From this observation, it can be concluded that MS in blended SCC mixtures imparts high strength while MK inclusion enhances the usage of high quantity of fly ash in SCC mixes for similar strengths and flow properties. The quaternary blended fly ash based M80 grade SCC mix made of MS and MK together is found to be superior to ternary blended fly ash based M80 grade SCC mix made with MS or MK due to reason that for similar strength, less cement is used and more fly ash quantity is consumed. Efficiency factor for quaternary blended SCC mix reveals that for similar strength, $50 \%$ of cement can be replaced with FA2 $2 \%+$ MS11 $\%+$ MK11\% combination of pozzolanic mixture.
\end{abstract}

Index terms - Self-compacting concrete, metakaolin, quaternary blended, efficiency factor, rheology, Compressive Strength.

\section{INTRODUCTION}

Though self-compacting concrete (SCC) can be used on most construction sites, its rheological characterization must be enhanced to better control its placement. Also, the fresh SCC must be stable to safeguard the homogeneity of the mechanical strength of the structure. The stability of SCC can be improved by incorporating fine materials such as metakaolin (MK), micro silica (MS) and fly ash (FA) because an increase in cement content leads to a substantial rise in material cost and often has other negative effects on concrete properties (e.g. increased thermal stress and shrinkage, etc.) [1].The use of such pozzolans may provide greater cohesiveness by improving the grain-size distribution and particle packing [2]. But the use of mineral admixtures not only reduced the material cost but also improved the fresh and hardened properties of blended SCCs [3].In recent years, there has been a growing attention in the use of metakaolin (MK) as a mineral admixture to enhance the properties of concrete [4].In the literature, however, the use of MK in the production of selfcompacting concrete has not found adequate interest. Considered to have high reactivity than most other pozzolans, metakaolin is a valuable admixture for concrete/cement applications [5]. Replacing Portland cement with $8-20 \%$ (by weight), metakaolin produces a concrete mix which exhibits favorable engineering properties, including: the filler effect, the acceleration of OPC hydration, and the pozzolanic reaction [6]. The filler effect and hydration reaction is immediate, while the effect of pozzolanic reaction occurs between 3 to 14 days.

\section{II.OHJECTIVE}

This study systematically investigate the synergistic effect of metakaolin (MK) and microsilica (MS) on fresh and strength properties of fly ash based SCC of M80 grade. These results are compared to establish the enhanced micro-structural and engineering properties of metakaolin based quaternary blended SCC over ternary blended SCC. The use of appropriately proportioned metakaolin and microsilica in fly ash blended SCC reveal the benefits of their synergic effect in improving the rheological properties, strength characteristics of fly ash based M80 grade SCC. The primary objectives of this research work is to quantitatively comprehend and assess the role of metakaolin (MK) in development of early strength in fly ash based SCC of High strength grade (M80). 


\section{Materials And Mix Proportions}

The materials used in the experimental investigation are locally available OPC cement, river sand (Zone -II), coarse aggregate $(10 \mathrm{~mm})$, mineral and chemical admixtures such as fly ash, micro silica and metakaolin, PCE based Super Plasticizer (SP), Viscosity Modifying Agent (VMA), Based on Nan Su mix design method, material quantities such as powder content (Cement + Pozzolan), fine aggregate, coarse aggregate, water and dosages of SP and VMA required for $1 \mathrm{cu.m}$, are evaluated for high strength grade (M80) of Self Compacting Concrete (SCC). Final mix proportions and optimum proportions of FA, MS and MK combinations in binary, ternary and quaternary blended high strength SCC mix which are assumed after several trial mixes on material quantities computed using Nan Su mix design method; subjected to satisfaction of EFNARC flow properties.

\section{IV, EXPERIMENTAL INVESTIGATIONS}

The aim of the present experimental investigations are aimed to obtain specific experimental data which helps to understand the effect of synergic action of metakaolin (MK), micro silica (MS) and fly ash (FA) combinations in SCC mixes of high strength grade (M80) on rheological behavior and strength properties. Test for Compressive strengths at $3,7,28,60$ and 90 days were determined by conducting detailed laboratory investigations on high strength grade (M80) for optimally blended self compacting Concrete (SCC) mixes made with fly ash (FA), microsilica (MS) and metakaolin (MK).For calculating the efficiency of metakaolin, microsilica and fly ash combination in quaternary blended SCC, an equation has been proposed by author based on the principle of Bolomey's equation for predicting the strength of concrete containing mineral admixtures. The efficiency factors evaluated can be used for proportioning of high strength grade (M80) of binary, ternary and quaternary blended selfcompacting concrete (SCC) made with $\mathrm{SCMs}$ such as fly ash (FA), microsilica (MS) and metakaolin(MK).

\section{Evaldation Of Cementing EFficiency Factors}

An effort is made to quantify the cementitious efficiency of fly ash (FA), microsilica (MS) and metakaolin (MK) in binary, ternary and quaternary blended self-compacting concrete (SCC) systems of high strength grade (M80). The effect of synergic action of metakaolin (MK), microsilica (MS) and fly ash (FA) combination on the strengths of binary, ternary and quaternary blended SCC may be modelled by using a Cementing Efficiency Factor (k).The concept of efficiency can be used for comparing the relative performance of SCMs when incorporated into SCC to the performance of OPC SCC. Efficiency factors found from Bolomey's strength equation are used to describe the effect of the SCMs combination replacement in SCC in the enhancement of strength characteristics. This factor will give only an indication of the added materials' effect on concrete strength, since it does not distinguish between filler effect and chemical reactions. The well-known Bolomey's equation often used to relate strength and watericement ratio is: $=\mathrm{A}[(\mathrm{C}+\mathrm{kP}) / \mathrm{W})-0.5]$ where $\mathrm{S}$ is the compressive strength in $\mathrm{MPa}, \mathrm{C}$ is the cement content in $\mathrm{kg} / \mathrm{m}^{3}, \mathrm{~W}$ is the water content in $\mathrm{kg} / \mathrm{m}^{3}, \mathrm{~A}$ and $\mathrm{B}$ are Bolomey's coefficients for constants, P is the amount of SCMs replaced bwc,k denotes efficiency factor of SCMs combination.By knowing the amounts of ' $\mathrm{C}$ ', ' $\mathrm{P}$ ', 'W' and the strength ' $\mathrm{S}$ ' achieved for each SCMs dosage replacement, efficiency factor " $\mathrm{k}$ " has been computed for each of the replacement dosages. Thus, W/(C+kP) is the water/effective powder ratio and $\mathrm{kP}$ is the equivalent cement content of SCMs combination. 'SCMs /OPC ratio' is an important factor for determining the efficiency of SCMs in SCC. So SCMs proportioning is arrived at based on the strength data experiments on SCMs blended SCC Mixes. Efficiency factors found from this strength equation are used to describe the effect of the SCMs replacement.

\section{TEST RESULTS AND DISCUSSIONS}

\section{A. Optimization of Mix Proportions}

The initial quantities calculated using Nan Su method for high strength (M80) grade SCC mix are tabulated in Table 1.The computed amount of total powder (i.e., OPC $+\mathrm{FA}$ ) is $658 \mathrm{~kg}$. For the above quantities, even though flow properties are achieved conforming to EFNARC guidelines, the high quantity of cement computed using Nan Su method is a matter of concern. From durability perspective, the maximum cement content is limited to $450 \mathrm{~kg}$ per cum of concrete as per clause 8.2 .4 .2 of IS $456-2000$. After trail mixes, revised quantities in $\mathrm{kg}$ per cu.m for high strength grade (M80) SCC mix are arrived by (i) limiting the Cement to maximum permissible amount, (ii) increasing quantity of pozzolan (fly ash) to maximum amount possible and adjusting the super plasticizer without compromising the EFNARC flow properties and desired strength property. The final revised quantities for high strength M80 grade SCC mix are tabulated in Table 2.

Henceforth, the total amount of powder quantity (cement + pozzolanic mixture) adopted for high strength M 80 SCC is $700 \mathrm{~kg} / \mathrm{m}^{3}$ and water/powder ratio is 0.25 for all blended high strength M80 SCC mixes. For higher grades, Nan Su mix design method computations yield very less powder content. In fact, from the observations it may be stated that Nan Su method is very difficult to apply for higher grades of concrete to arrive at appropriate quantities of materials. Depending on the above calculated base quantities for high strength grade (M80), twenty nine (29) blended SCC mixes were designed in three groups of binary, ternary and quaternary. Table 3 shows various blended high strength grade (M80) SCC mixtures with mix numbers and mix designations. In mix designation, number indicates percentage by weight of total powder content. One reference SCC mix was also prepared by only OPC (Mix C1) while in the remaining mixtures (Mix B1 to B8, Mix $\mathrm{T} 1$ to $\mathrm{T} 8$ and Mix Q1 to Q12) OPC was partially replaced with fly ash (FA), microsilica (MS), metakaolin (MK) and their combinations. Mix B1 to B8 are binary blended SCC mixtures made of either fly ash (FA) or microsilica (MS) or metakaolin (MK) while Mix T1 to T8 are ternary blended fly ash based SCC mixtures made of microsilica (MS) or metakaolin (MK) and Mix Q1 to Q12 are quaternary blended fly ash based SCC mixtures made of microsilica 
(MS) and metakaolin (MK) together. In binary blended high strength grade (M80) SCC mixtures, percentage replacement of fly ash by weight of total powder content is $35 \%$ i.e. $250 \mathrm{~kg} / \mathrm{m}^{3}$ which is based on preliminary calculation from mix design method. For binary blended SCC mixtures made with percentage replacement of MS or MK, MS and MK are limited to $5-15 \%$ and $5-20 \%$ respectively, In ternary blended MS+FA based high strength grade (M80) SCC mixtures (Mix T1 to T4) percentage replacement of MS is limited to $5-20 \%$ by weight of total powder content. Similarly in ternary blended $\mathrm{MK}+\mathrm{FA}$ based high strength grade (M80) SCC mixtures (Mix T5 to T8), percentage replacement of MK is limited to $5-20 \%$ by weight of total powder content. In both the above ternary blended MS+FA based $\mathrm{SCC}$ and $\mathrm{MK}+\mathrm{FA}$ based SCC mixtures (Mix T1 to T8), the cement content is kept constant ( $65 \%$ by weight of total powder content). In high strength grade (M80) SCC mix $\mathrm{Cl}$ developed with $100 \%$ OPC does not yield desired strength though required flow properties are achieved. In binary blended high strength grade (M80), FA based SCC mix (Mix B1) and MK based SCC mixes (Mix B5 to B8), although required flow properties are achieved but desired strength is not realized while in binary blended MS based SCC mixes, both required flow properties and desired strength are attained, if the MS percentage replacement is limited to 5$10 \%$ by weight of powder. The optimal mix chosen for binary blended MS based SCC mix is $5 \%$ MS replacement (Mix B2). Henceforth, for high strength grade (M80) mixes, Mix B2 is taken as reference mix. In ternary blended $\mathrm{MK}+\mathrm{FA}$ based high strength grade (M80) SCC mixtures (Mix T5 to T8), however required flow properties are satisfied but desired strengths are not obtained for any of the mixes. But for MS+FA based ternary blended SCC mixes ( $\mathrm{T} 1$ toT4), up to $15 \%$ MS by weight of powder, both required flow properties and desired strength are attained satisfactorily. So C65+FA20+MS15 (Mix T3) SCC mix is considered optimal in ternary blended high strength grade (M80) SCC mixes, In quaternary blended high strength grade (M80) SCC mixtures (Mix Q1 to Q12) made of microsilica (MS), metakaolin (MK) together, keeping cement content constant ( $65 \%$ by weight of total powder content), MS and MK contents are limited to $7-14 \%$. For quaternary blended SCC mix (Mix Q1), initially $7 \%$ MS and $7 \%$ MK replacements are assumed, keeping cement content constant i.e. $65 \%$ by weight of total powder content and rest of powder is fly ash, required flow properties are satisfied but desired strengths are not obtained. So MS and MK are gradually increased to $14 \%$ each yet there is no substantial increase in strength though flow properties are satisfied. Then author proposed to additionally increase fly ash content incrementally by $10 \%$ by weight of powder content $(700 \mathrm{~kg} / \mathrm{m} 3)$, thereby incrementally increasing the powder quantity by $70 \mathrm{~kg}$. The optimum combination of cement and pozzolanic mixture is obtained for C50+FA28+MS11+Mk11 SCC mix (Mix Q11) where final total powder content is $910 \mathrm{~kg} / \mathrm{m} 3$ in which cement content is $455 \mathrm{~kg} / \mathrm{m} 3$ and pozzolanic mixture is $455 \mathrm{~kg} / \mathrm{m} 3$. For this optimum mix (Mix Q11), MS and MK are optimally proportioned at $14 \%$ each and additional percentage of FA is $30 \%$ by weight of powder content $(700 \mathrm{~kg} / \mathrm{m} 3)$, for which required flow and strength properties are fulfilled. From table 3, three optimum SCC mixes are nominated, one each from binary, ternary and quaternary SCC blends. From the experimental investigations, the mixes B2, T3 and Q11 are chosen as optimum binary, ternary and quaternary blended high strength grade (M80) SCC mixes where both flow and desired strength properties are met along with optimal usage of pozzolanic quantities as shown in Table 4 and 5 . Thus, by incorporating MK into MS+FA based ternary blended SCC mixes, the amount of fly ash has almost doubled. From this observation, it can be understood that MS in blended SCC mixtures imparts high strength while MK inclusion enhances the usage of high quantity of fly ash in SCC mixes for similar strengths and flow properties. The quaternary blended fly ash based SCC mix made of MS and MK together is found to be superior to ternary blended fly ash based SCC mix made with MS or MK due to reason that for similar strength, less cement is used and more fly ash quantity is consumed to develop blended high strength grade (M80) SCC.

Based on the compressive strength attained at specified age of curing, the efficacy of pozzolans are understood. In this study, pozzolans used for blended SCC mixes are FA, MS and MK. MK blended fresh concretes will set relatively quickly due to high reactivity of MK, which prevents bleeding and settling of aggregates.MK when compared to MS has similar particle density and surface area but different morphology and surface chemistry. MK concrete normally requires smaller SP dose than does the equivalent SF concrete. The workability of FA based SCC concrete, without super plasticizer, increases significantly with increase in FA content due to neutralization of positive charges on cement particles and their resultant dispersal. Loss of workability due to the presence of MK or MS can be compensated for by the incorporation of FA. The degree of restoration of workability, provided by FA, is influenced significantly by the cement replacement level, the MK/FA ratio and the $\mathrm{W} / \mathrm{P}$ ratio. 


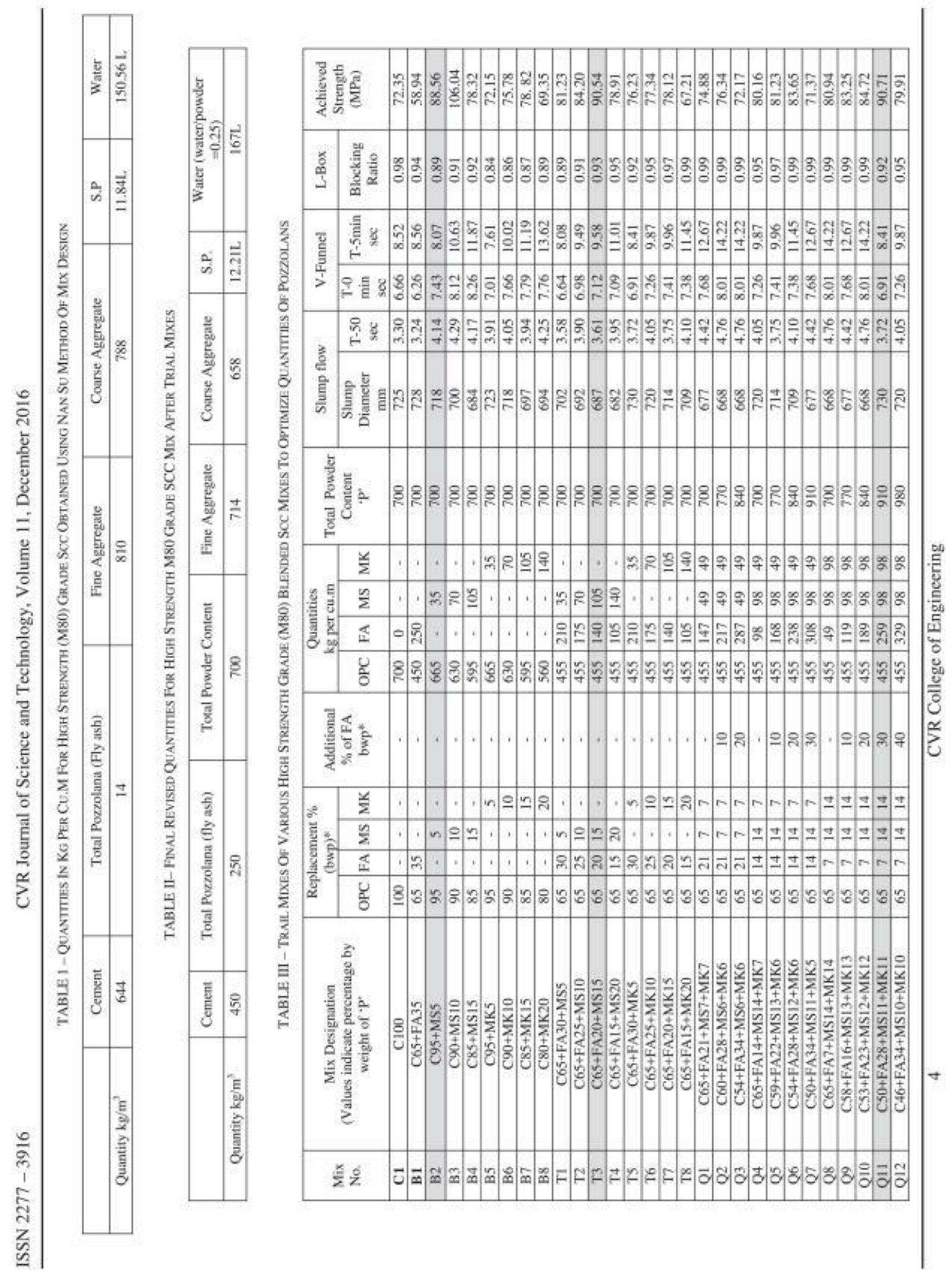




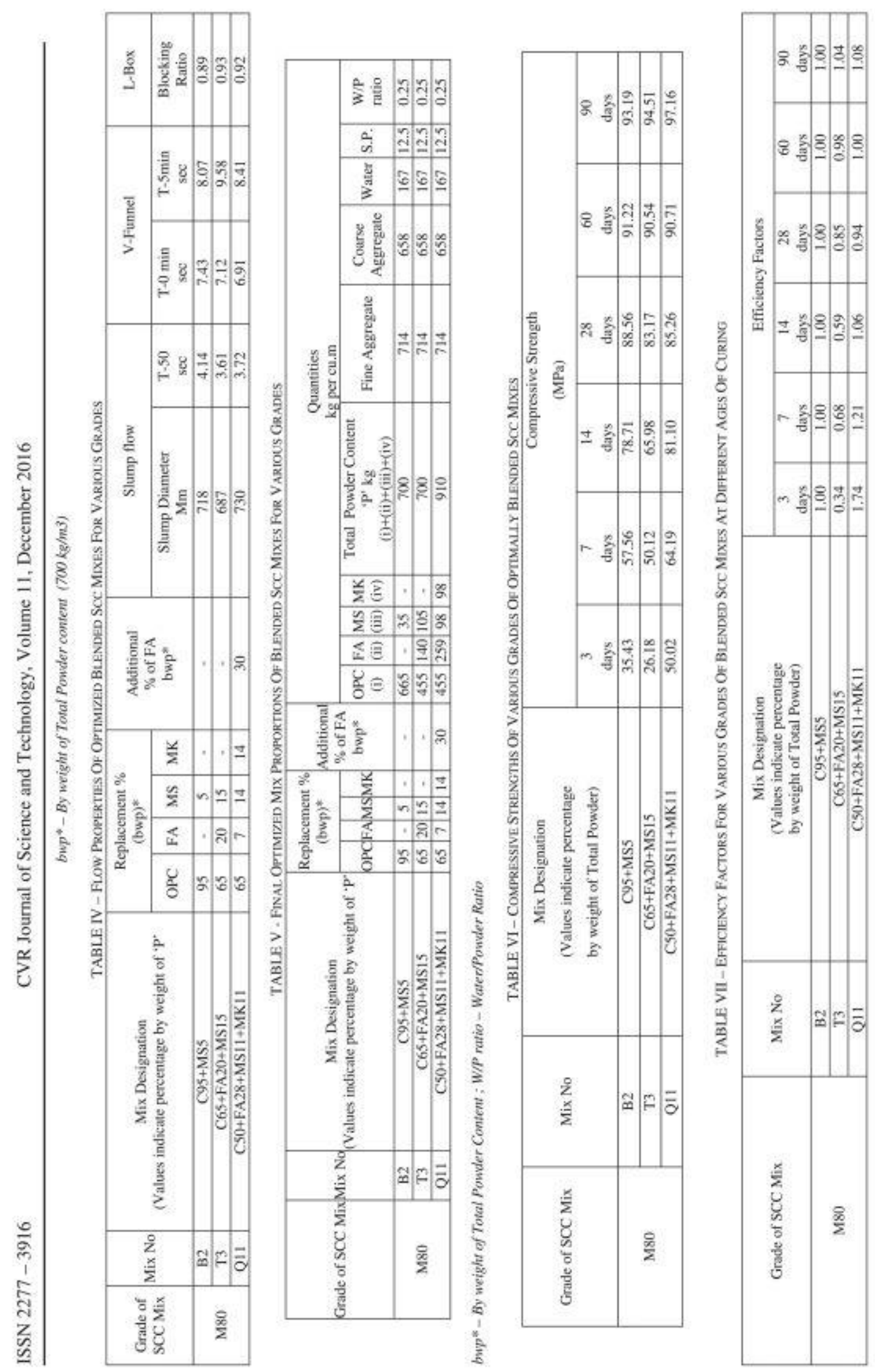




\section{B. Compressive Strength}

This investigation is carried out to study the compressive strength of binary, ternary and quaternary blended SCC mixes of high strength grade (M80) made with fly ash (FA), microsilica (MS) and metakaolin (MK) at 3,7,14,28, 60 and 90 days.Table 6 presents the compressive strength at various ages of curing for binary, ternary and quaternary blended SCC mixes of high strength grade (M80). It can be observed that there is no much variation in strength at the stipulated age of curing, in all SCC mixes as shown in Figure 1.MK based blended SCC mixes attain much higher early strength when compared to other SCC mixes while FA based SCC mixes are accomplishing strengths at later age. So MK+FA based SCC blends derive both the benefits of FA and $\mathrm{MK}$ in concrete by attaining early and later strengths. Use of MS in high strength grade (M80) acts as a micro-filler in making the concrete dense. Metakaolin cementing reaction rate is very rapid, significantly increasing compressive strength before first three days. Also MK being less expensive than MS when incorporated into MS+FA based ternary blended SCC mixes, the amount of fly ash used has almost doubled. From this observation. it can be understood that MS in blended SCC mixtures imparts high strength while MK inclusion enhances the usage of high quantity of fly ash in SCC mixes for similar strengths and flow properties. Addition of MK to binary or ternary blended SCC mixes will enhance early hydration because of it high reactivity.

\section{Cementing Efficiency}

This study understands the behavior of MK in $\mathrm{FA}+\mathrm{MS}+\mathrm{MK}$ SCC mix in order to quantify the strength efficiency of FA+MS+MK in SCC at 3,7, 14,28,60 and 90 days. This evaluation makes it possible to design FA+MS+MK SCC for a desired strength at any given percentage of replacement. The strength efficiency factor ' $\mathrm{k}$ ' is evaluated for three cases: (1) MS is singly blended in SCC, (2) MS is blended with FA in SCC and (3) MK is blended with MS+FA in SCC. Based on the C95+MS5 compressive strength as reference, ' $\mathrm{A}$ ' value was calculated using Bolomey equation and then efficiency factors for $\mathrm{FA}+\mathrm{MS}+\mathrm{MK}$ and $\mathrm{FA}+\mathrm{MS}$ in $\mathrm{SCC}$ mixes were then determined using same Bolomey's equation. From table 7, it can be observed that efficiency factors for binary (Mix B2), ternary (Mix T3) and quaternary (Mix Q11) blended high strength SCC mixes are 1.00, 1.04 and 1.08 respectively. All the three M80 grade $\mathrm{SCC}$ mixes give similar strength and satisfy EFNARC specifications. The efficiency factor for quaternary (C50+FA28+MS11+MK11) blended high strength SCC mixes is 1.08 which means that $1 \mathrm{~kg}$ of cement can be replaced with $1.08 \mathrm{~kg}$ of FA+MS+MK pozzolanic mixture.

\section{Conclusions}

Based on the test results and discussions, the following conclusions are drawn:

1. For binary and ternary blended $\mathrm{SCC}$ mixtures made with percentage replacement of MS or MK, MS and MK are limited to $5-15 \%$ and $5-20 \%$ respectively.
2. In quaternary blended high strength grade (M80) SCC mixtures made of microsilica (MS) and metakaolin (MK) together, keeping cement content constant ( $65 \%$ by weight of total powder content), MS and MK contents are limited to $7-14 \%$.

3. Incorporating $\mathrm{MK}$ into $\mathrm{MS}+\mathrm{FA}$ based ternary blended SCC mixes, the amount of fly ash has almost doubled. From this observation, it can be understood that MS in blended SCC mixtures imparts high strength while MK inclusion enhances the usage of high quantity of fly ash in SCC mixes for similar strengths and flow properties.

4. The quaternary blended fly ash based SCC mix made of MS and MK together is found to be superior to ternary blended fly ash based SCC mix made with MS or MK due to reason that the less cement is used and more fly ash quantity is consumed to develop blended high strength grade (M80) SCC of same strength. Also Quaternary blended fly ash based $\mathrm{SCC}$ mix giver better early strength than ternary blended SCC mix.

5. From this observation, it can be understood that MS in blended SCC mixtures imparts high strength while MK inclusion enhances the usage of high quantity of fly ash in SCC mixes for similar strengths and flow properties.

6. Efficiency factor for quaternary blended SCC mix reveals that for similar strength, $50 \%$ of cement can be replaced with FA28 $\%+\mathrm{MS} 11 \%+\mathrm{MK} 11 \%$ combination of pozzolanic mixture.

\section{REFERENCES}

[1]. ErhanGuneyisi \& Mehmet Gesoglu 2008, 'Properties of self- compacting mortars with binary and ternary cementitious blends of fly ash and metakaolin', Materials and Structures, Vol. 41, no. 9,pp. 1519-1531.

[2]. Mousavi, S 2012, 'Fresh and hardened properties of self-compacting concrete containing metakaolin', Construction and Building Materials, Vol. 35, no. 1, pp. $752-760$.

[3]. Jiping BAI, Stan Wild and AlbinasGailius2004, 'Accelerating Early Strength Development of Concrete Using Metakaolin as an Admixture', Materials Science, Vol. 10, no. 4, pp. 338-344.

[4]. Melo, KA andCarneiro, AMP 2010, 'Effect of Metakaolin's finesses and content in self consolidating concrete', Construction Building Materials, Vol. 24, pp.1529-1535.

[5]. Poon, CS, Lam, I, Kou, SC, Wong, YL and Wong, R 2001 , 'Rate of pozzolanic reaction of metakaolin in high-performance cement pastes', Cement and Concrete Research, Vol. 31, no. 9, pp. 1301-1306.

[6] Sabir, BB, Wild, S and Bai, J 2001, 'Metakaolin and Calcined Clays as Pozzolans for concrete: A Review', Cement and Concrete Composites, Vol. 23, no. 6, pp. 441-454. 